\title{
Implementação de novas práticas ou como mudar comportamentos ${ }^{1}$
}

José Mendes Nunes*

\section{RESUMO}

Entende-se por Desenvolvimento Profissional Contínuo (DPC) o processo de aprendizagem ao longo da vida, para todos os profissionais e equipas, que visa satisfazer as necessidades dos doentes, com impacto nos resultados em saúde, e nas prioridades do sistema de saúde, para além de permitir a expansão e realização do potencial dos profissionais. Neste processo de aprendizagem, até ao momento, a ênfase tem sido na aquisição de conhecimentos. No entanto, o objectivo é modificar os comportamentos na prática de prestação de cuidados de saúde e, tal como a prática clínica, deve ser fundamentada pela Medicina Baseada na Evidência (BEM). A incorporação desta na actividade diária, deve ser sustentada por estratégias que demonstraram ser efectivas na mudança dos comportamentos, isto é, deve recorrer a práticas baseadas na evidência.

Palavras-chave: Desenvolvimento Profissional Contínuo; Mudança de Comportamentos.

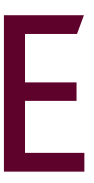

m termos de Desenvolvimento Profissional Contínuo (DPC) dá-se grande ênfase à aquisição de conhecimentos, para não dizer que na maioria das situações se reduz apenas a isso.

Contudo, o grande desafio no DPC está na capacidade de modificar os comportamentos na prática prestadora de cuidados. Em geral, a formação é determinada porque está disponível ou porque alguém induziu a sua necessidade, seja por interesses de negócio, de currículo, de prestígio ou por gosto pessoal. As acções de formação devem estar associadas às necessidades identificadas na sequência de avaliação das actividades. Os planos de DPC devem ser determinados, apenas e só, pelas necessidades de cuidados da população.

Grande parte da actividade formativa enferma de mitos como: «quem sabe é competente»e «quem é competente tem bom desempenho». Temos conceitos diferentes que são confundidos: ter conhecimentos não significa que os seja capaz de aplicar; quem é capaz de os aplicar não quer dizer que os aplique. Há, então, di-

\footnotetext{
*Assistente Graduado Sénior da USCP de Almeirim (ACES da Lezíria). Assistente convidado do Departamento de MGF, da Faculdade de Ciências Médicas de Lisboa, Universidade Nova de Lisboa.

${ }^{1}$ Texto redigido no âmbito do trabalho do Projecto de Apoio à Criação e Desenvolvimento dos Conselhos Clínicos dos ACES da ARSLVT, IP.
}

ferença entre conhecimento, competência e desempenho.

Por outro lado, a maioria das acções formativas têm subjacente a ideia de que o inadequado desempenho se deve à falta de conhecimentos. Como constantemente se verifica que o desempenho não é o concordante com a Medicina Baseada na Evidência (MBE), então a conclusão será: «é preciso formação». Assim, mantém-se uma intensa (e onerosa) actividade formativa, onde muitos ensinam mas poucos aprendem, resultante do ciclo: inadequado desempenho, exige formação, mantém-se inadequado desempenho, então, é preciso mais formação. Assim, perante a constatação de que os profissionais não mudam o comportamento, faz-se «mais do mesmo»: mais formação.

Nestas circunstâncias, a implementação da MBE deve ser acompanhada de actividades formativas que tenham em consideração que o seu objectivo é a modificação de comportamentos. Enquanto as populações não usufruírem dos conhecimentos técnico-científicos a formação ou educação profissional é socialmente inútil. Tal como a prática clínica deve ser fundamentada pela $\mathrm{MBE}$, a incorporação desta na prática clínica, deve ser sustentada por estratégias que demonstraram ser efectivas na mudança dos comporta- 
mentos, isto é, deve recorrer a práticas baseadas na evidência. ${ }^{1}$ Por outras palavras, associado ao conhecimento do que deve ser feito deve existir uma actualização sobre o modo como levar os profissionais $a$ fazer. Não existem estratégias milagre para a mudança de comportamentos clínicos, pelo que o mais adequado é utilizar várias estratégias, de acordo com o contexto do que se pretende mudar (o quê, quem, quando, onde).

\section{ESTRATÉGIAS PARA DISSEMINAÇÃO E IMPLEMENTAÇÃO DA MBE}

\section{Educação Médica Contínua}

Envolve um largo espectro do conhecimento não se dirigindo a um comportamento específico. Dentro destas metodologias incluem-se: encontros, conferências, lições, sessões interactivas (workshops), seminários, cursos e simpósios. De todas estas modalidades, só as acções de formação interactivas (workshops) parecem provocar modificação significativa na prática profissional. Já as sessões didácticas (ex-catedra) parecem ter um impacto muito reduzido na alteração de comportamentos. ${ }^{2}$ No entanto, o recurso a metodologias diversificadas, particularmente se as potenciais barreiras foram previamente identificadas, aumenta a probabilidade de sucesso na mudança da prática.

\section{Introdução de Normas de Orientação Clínica (NOC)}

A disseminação passiva de NOCs modifica muito pouco os comportamentos. A resistência à implementação de uma NOC é tanto maior quanto maior for a sua complexidade. ${ }^{6}$ e $^{3}$ No entanto, existem alguns factores que podem favorecer a implementação de uma NOC:

- Ter em consideração as circunstâncias locais;

- Ser divulgada conjuntamente com intervenções educacionais activas;

- Acompanhar a implementação com recordatórios específicos de doente, i.e., o recordatório surge sempre que o sistema informático identifica uma situação em que a norma deve ser aplicada;

- Ser produzida pelos próprios ou, pelo menos, com a sua colaboração.

\section{Disseminação de materiais educacionais}

Não há evidência de que a disseminação passiva de material educacional seja efectiva na mudança de comportamentos. ${ }^{4} \mathrm{Um}$ estudo em que se reviram 11 ensaios não revelou efectividade. ${ }^{5}$ Outro trabalho, com 18 estudos, verificou que o efeito é muito efémero (citado por Van Zwanenberg ${ }^{6}$ ). No entanto, deve-se ter em consideração que estes materiais são fundamentais para se associarem a outras metodologias. O problema é que a sua divulgação é muitas vezes entendida como «está dito, está feito»e não é dada continuidade com outras metodologias. Os materiais educacionais devem ser entendidos como instrumentos de apoio para as diferentes acções de formação e para a colocação do respectivo tema na agenda formativa.

\section{Visitas educacionais}

Definidas como o uso de pessoa treinada que se encontra com o profissional no seu setting de trabalho e fornece-lhe informação com o objectivo de mudança. A informação fornecida pode incluir retroinformação sobre o desempenho pessoal ou sobre como ultrapassar os obstáculos que se opõem à mudança. Parece ser um método efectivo na modificação dos comportamentos de prescrição, mas já não tem tanto impacto noutras vertentes da prática, como seja a utilização de testes de rastreio ou de diagnóstico. ${ }^{7}$

\section{Líderes locais}

Consiste na utilização de prestadores percepcionados pelos pares como influentes. É uma metodologia que só foi testada para a prática hospitalar. ${ }^{8} \mathrm{~Pa}-$ rece ser efectiva na promoção das práticas baseadas na evidência, particularmente, se forem os profissionais alvo a designar quem os lidere, no entanto, tem o problema de não ser muito exequível na prática.

6. Formação multiprofissional

A formação interprofissional oferece uma oportunidade de melhoria das práticas. Alguns estudos parecem mostrar efectividade da educação multiprofissional nos departamentos de emergência, na melhoria da satisfação dos utentes, na diminuição dos erros e na prestação de cuidados a vítimas de vio-

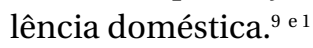

\section{Auditoria com retro-informação}

Consiste na auditoria à prática, seguida de apresentação dos respectivos resultados. Pode influenciar a prescrição e a requisição de MCDT, no entanto, o efeito é modesto e os estudos realizados têm grande 
variabilidade no que se entende por auditoria e retro-informação. A sua efectividade é tanto maior quando for menor o desempenho de base. ${ }^{11}$

\section{Uso de recordatórios}

Apesar de tudo é a estratégia mais efectiva, particularmente se for associado a sistemas de decisão computorizados. De qualquer modo, o seu efeito nota-se sobre a prescrição e na prevenção mas não no diagnóstico. ${ }^{12}$ Este é mais um aspecto que põe em relevo a importância do desenvolvimento e implementação dos sistemas informáticos. Estes devem obedecer ao princípio de que se destinam prioritariamente a suportar a actividade de prestação de serviços, ao contrário do que se tem verificado até ao momento, em que a prioridade é fornecer informação para a gestão.

\section{ESCOLHA DE ESTRATÉGIAS PARA DISSEMINAÇÃO E IMPLEMENTAÇÃO DA MBE}

A escolha da estratégia para a implementação de uma NOC (pressupõe-se que fundamentada na MBE) passa pela identificação das eventuais barreiras que se opõem à mudança de comportamento. ${ }^{13}$

Na base dos obstáculos à mudança de comportamento podem estar:

- Dificuldades em reconhecer a importância da mudança;

- Barreiras culturais que se opõem à mudança;

- Incapacidade de processar múltipla informação.

Seja como for, neste contexto, aplicam-se os requisitos básicos para a mudança de comportamentos:

- Reconhecimento da importância de mudar;

- Percepção da auto-eficácia para a mudança.

Assim, a escolha de uma estratégia para a mudança de comportamento deve ter em consideração (Figura 1):

- Identificar as actividades alvo (o que deve mudar);

- Identificar os profissionais alvo (quem deve mudar);

- Identificar as barreiras que se opõem à mudança (obstáculos à mudança).

Em resumo, a implementação das recomendações de NOCs exigem o planeamento de estratégias fundamentadas em alguns princípios.

1. Não basta fornecer formação ou informar, é imprescindível que se associem estratégias que aumentem a probabilidade de o conhecimento se reflectir na prática. Qualquer NOC ou acção de formação tem como objectivo modificar a prática; a transferência de informação é um meio mas não o fim.

2. As NOCs devem ser simplificadas ao máximo. A dispersão da atenção por várias mudanças em simultâneo reduz a probabilidade de êxito.

3. As estratégias de mudança de comportamentos devem ser diversificadas e utilizadas em simultâneo de modo a potenciarem-se. As estratégias multifacetadas dirigidas a um comportamento específico são as que têm maior probabilidade de sucesso.

4. Não há aderência à mudança sem o reconhecimento de que é importante mudar. Criar a necessidade de mudar.

5. Há que criar condições para que quem muda percepcione que é capaz de dar o salto qualitativo.

6. Num processo de mudança deve-se estar atento aos obstáculos que se lhe opõem, nomeadamente não «matar o mensageiro», isto é, ignorar os indícios que apontam para os obstáculos percepcionados pelos executantes.

7. Os sistemas de informação são instrumentos vitais no apoio à mudança fornecendo retro-informação, em tempo real, transmitindo segurança e emitindo recordatórios (red flags) associados aos actos ou aos doentes.

8. A mudança deve ser reconhecida, com reforço condicional positivo desde os primeiros índices mínimos de mudança.

9. Na implementação de recomendações, ninguém muda de repente e nem todos mudam ao mesmo tempo.

10. No planeamento da implementação de recomendações para a prática, ter em consideração que as mudanças são graduais e progressivas, evitando querer mudar tudo ao mesmo tempo e mais do que o possível. Atenção: o desejável pode estar muito além do possível.

\section{REFERÊNCIAS BIBLIOGRÁFICAS}

1. Department of Health. A First-Class Service: quality in the new NHS. London: Department of Health; 1998. Disponível em: http://www.dh.gov.uk/en/publicationsandstatistics/publications/publicationspolicyandguidance/dh_4006902 [acedido em 31/10/2010]. 
2. Thomson O'Brien MA, Freemantle N, Oxman AD, Wolf F, Davis D, Herrin J. Continuing education meetings and workshops: effects on professional practice and health care outcomes. Cochrane Database Syst Rev 2001; (2): CD003030.

3. McSherry R, Pearce P. Clinical Governance: a Guide to Implementation for Healthcare Professionals. Malden, MA: Blackwell Publishing; 2007.

4. Farmer AP, Légaré F, Turcot L, Grimshaw J, Harvey E, McGowan JL, Wolf F. Printed educational materials: effects on professional practice and health care outcomes. Cochrane Database of Systematic Reviews 2008 , Issue 3.Art. No.: CD004398. DOI: 10.1002/14651858.CD004398.pub2.

5. Farmer AP, Légaré F, Turcot L, Grimshaw J, Harvey E, McGowan JL, et al. Printed educational materials: effects on professional practice and health care outcomes. Cochrane Database Syst Rev 2008 Jul 16; (3): CD004398.

6. Van Zwanenberg T, Harrison J. Clinical Governance in Primary Care. 2nd ed. Abingdon, Oxon: Radcliffe Medical Press; 2007. p. 80.

7. O'Brien MA, Rogers $S$, Jamtvedt $G$, Oxman AD, Odgaard-Jensen J, Kristoffersen DT, et al. Educational outreach visits: effects on professional practice and health care outcomes. Cochrane Database Syst Rev 2007 Oct 17; (4): CD000409.

8. Doumit G, Gattellari M, Grimshaw J, O'Brien MA. Local opinion leaders: effects on professional practice and health care outcomes. Cochrane Database Syst Rev 2007 Jan 24; (1): CD000125.

9. Reeves $\mathrm{S}$, Zwarenstein $\mathrm{M}$, Goldman J, Barr H, Freeth $D$, Hammick $M$, et al. Interprofessional education: effects on professional practice and health care outcomes. Cochrane Database Syst Rev 2008 Jan 23; (1) CD002213.

10. Baker DP, Day R, Salas E. Teamwork as an essential component of highreliability organizations. Health Serv Res 2006 Aug; 41 (4 Pt 2): 157698.

11. Jamtvedt G, Young JM, Kristoffersen DT, O'Brien MA, Oxman AD. Audit and feedback: effects on professional practice and health care outcomes. Cochrane Database Syst Rev 2006 Apr 19; (2): CD000259.

12. Hunt DL, Haynes RB, Hanna SE, Smith K. Effects of computer-based clinical decision support systems on physician performance and patient outcomes: a systematic review. JAMA 1998 Oct 21; 280 (15): 133946.

13. Shaw B, Cheater F, Baker R, Gillies C, Hearnshaw H, Flottorp S, et al. Tailored interventions to overcome identified barriers to change: effects on professional practice and health care outcomes. Cochrane Database Syst Rev 2005 Jul 20; (3): CD005470.

\section{CONFLITOS DE INTERESSE}

O autor declarou não possuir conflitos de interesses.

\section{ENDEREÇO PARA CORRESPONDÊNCIA}

jmnunes@netcabo.pt

Recebido em 01/10/2009

Aceite para publicação em 05/11/2009

\begin{abstract}
IMPLEMENTATION OF NEW OR CHANGING PRACTICE BEHAVIORS

By Continuous Professional Development, we mean a long life process of learning for all professionals and teams, with the aim to satisfy the patients' health needs, with impact on health outcomes, and health systems priorities, respecting the expansion and fulfilment of professionals' potential. In this learning process, till the moment, the great emphasis has been on acknowledge acquisition. However, the aim is to change the behaviour in health care practice and it must be asserted on Evidence Based Medicine. The implementation of this activity in daily-practice must be sustained by strategies that have been demonstrated to be effectives for behaviours change, that is, must appeal for practice evidence based.
\end{abstract}

Keywords: Continuous Professional Development; Behaviour change. 